WUGRAV 92-14

\title{
Spin effects in the inspiral of coalescing compact binaries
}

\author{
Lawrence E. Kidder and Clifford M. Will \\ McDonnell Center for the Space Sciences, Department of Physics \\ Washington University, St. Louis, Missouri 63130 \\ Alan G. Wiseman \\ Department of Physics and Astronomy \\ Northwestern University, Evanston, Illinois 60208
}

\begin{abstract}
We derive the contributions of spin-orbit and spin-spin coupling to the gravitational radiation from coalescing binary systems of spinning compact objects. We calculate spin effects in the symmetric, trace-free radiative multipoles that determine the gravitational waveform, and the rate of energy loss. Assuming a balance between energy radiated and orbital energy lost, we determine the spin effects in the evolution of the orbital frequency and orbital radius. Assuming that a laser interferometric gravitational observatory can track the gravitational-wave frequency (twice the orbital frequency) as it sweeps through its sensitive bandwidth between about 10 $\mathrm{Hz}$ and one $\mathrm{kHz}$, we estimate the accuracy with which the spins of the component bodies can be determined from the gravitational-wave signal.
\end{abstract}

04.30.+x,04.80.+z

Typeset Using REVTEX 


\section{INTRODUCTION}

The ability of laser interferometric gravitational-wave (GW) detectors such as the US LIGO to extract useful astrophysical information about coalescing binary systems of compact objects from the observed gravitational-wave signals depends upon highly accurate theoretical models for the decaying orbital evolution. Detection and study of the characteristic "chirp" waveform emitted by such systems involves a matched filtering technique using a theoretical template that is a function of parameters of the source [1]. As has been pointed out by Cutler et al. [2], matching the theoretical variation of the GW frequency with the data as the frequency sweeps through the detector bandwidth between 10 and $1000 \mathrm{~Hz}$ may be the most promising way to obtain estimates of the masses and spins of the bodies. To this end, one needs a theoretical formula for the evolution of the GW frequency that includes all dependence on the two masses and spins, and that is sufficiently accurate that errors in the model lead to cumulative phase errors no larger than $2 \pi$ radians over the potentially thousands of cycles observed in the frequency bandwidth of interest (larger phase errors substantially reduce the signal-to-noise of the matched filter).

Cutler et al. [2] have suggested that a model for the frequency evolution that is based on a post-Newtonian approximation may be inadequate unless the approximation is carried to extremely high orders beyond the lowest-order Newtonian and post-Newtonian corrections that have been derived to date [3,4]. In addition, they point out that the effects of spin-orbit and spin-spin coupling of the bodies have not been derived, even in the first post-Newtonian approximation. It is the purpose of this paper to remedy the latter situation.

We derive the spin-orbit and spin-spin contributions to the symmetric trace-free (STF) multipoles that enter the gravitational waveform for two-body systems, using a multipole formalism developed by Blanchet, Damour and Iyer (BDI) [5,6]. In addition to explicit spin

terms, the multipole formalism involves several time-derivatives of multipole moments that lead to expressions involving the two-body acceleration; to evaluate these moments consistently, we use post-Newtonian equations of motion that also include spin terms. Using these 
multipoles, we then evaluate the rate of energy loss from the binary system. Specializing to a circular orbit, we calculate the rate of decrease of orbital radius and the rate of change of orbital frequency, assuming energy balance and using an expression for energy that includes spin terms. Finally, we estimate the size of spin-orbit and spin-spin effects for coalescing binary systems of neutron stars and/or black holes on the accumulation of GW phase in a LIGO-type detector.

\section{EQUATIONS OF MOTION}

Equations of motion for bodies of arbitrary mass and spin have been developed by numerous authors (for reviews and references see [7 9]). For our purposes, only the Newtonian, spin-orbit (SO) and spin-spin (SS) terms will be needed; post- and post-post-Newtonian non-spin terms have been considered elsewhere [4. Although the inclusion of spin implies that we are treating extended bodies, we ignore tidal and quadrupole-coupling effects (even though rotationally induced quadrupole effects are proportional to (spin) ${ }^{2}$ ); for binary systems containing neutron stars or black holes, these are expected to be small until the very latest stage of inspiral and coalescence, except possibly for rapidly rotating Kerr black holes [10].

By eliminating the center of mass of the system, we convert the two-body equations of motion to an effective one-body equation of motion given by $(G=c=1)$

$$
\mathbf{a} \equiv \mathbf{a}_{\mathbf{1}}-\mathbf{a}_{\mathbf{2}}=-\frac{m}{r^{2}} \hat{\mathbf{n}}+\mathbf{a}_{\mathbf{S O}}+\mathbf{a} \mathbf{S S}
$$

where

$$
\begin{gathered}
\mathbf{a}_{\mathbf{S O}}=\frac{1}{r^{3}}\{6 \hat{\mathbf{n}}[(\hat{\mathbf{n}} \times \mathbf{v}) \cdot(\zeta+\xi)]-[\mathbf{v} \times(4 \zeta+3 \xi)]+3 \dot{r}[\hat{\mathbf{n}} \times(2 \zeta+\xi)]\} \\
\mathbf{a}_{\mathbf{S S}}=-\frac{3}{\mu r^{4}}\left\{\hat{\mathbf{n}}\left(\mathbf{S}_{\mathbf{1}} \cdot \mathbf{S}_{\mathbf{2}}\right)+\mathbf{S}_{\mathbf{1}}\left(\hat{\mathbf{n}} \cdot \mathbf{S}_{\mathbf{2}}\right)+\mathbf{S}_{\mathbf{2}}\left(\hat{\mathbf{n}} \cdot \mathbf{S}_{\mathbf{1}}\right)-5 \hat{\mathbf{n}}\left(\hat{\mathbf{n}} \cdot \mathbf{S}_{\mathbf{1}}\right)\left(\hat{\mathbf{n}} \cdot \mathbf{S}_{\mathbf{2}}\right)\right\}
\end{gathered}
$$

where $m=m_{1}+m_{2}, \mu=m_{1} m_{2} / m, r=|\mathbf{x}|, \mathbf{x}=\mathbf{x}_{\mathbf{1}}-\mathbf{x}_{\mathbf{2}}, \hat{\mathbf{n}}=\mathbf{x} / r, \mathbf{v}=\mathbf{v}_{\mathbf{1}}-\mathbf{v}_{\mathbf{2}}, \zeta=\mathbf{S}_{\mathbf{1}}+\mathbf{S}_{\mathbf{2}}$, $\xi=\left(m_{2} / m_{1}\right) \mathbf{S}_{\mathbf{1}}+\left(m_{1} / m_{2}\right) \mathbf{S}_{\mathbf{2}}$, and $\dot{r}=(\hat{\mathbf{n}} \cdot \mathbf{v})$. 
It is useful to note that spin-orbit and spin-spin effects are of order $(R / r) v \bar{v}$ and $(R / r)^{2} \bar{v}^{2}$, respectively, compared to the Newtonian acceleration, where $R$ and $\bar{v}$ denote the characteristic radius and rotation velocity of each body; these terms thus are formally of firstpost-Newtonian order. However, for compact bodies, $R$ is of order of $m$ or a few times $m$, and $\bar{v}$ could be of order unity, so that in practice these terms can be considered to be of post $^{3 / 2}$-Newtonian and post-post-Newtonian order, respectively (indeed they are so denoted in 2]).

Equations for the precession of the spins caused by spin-orbit (geodetic precession) and spin-spin (Lense-Thirring) couplings [0] 9] can also be written down in terms of relative coordinates. The relevant equation for our purposes is

$$
\dot{\zeta}=\frac{1}{r^{3}} \mathbf{L}_{\mathbf{N}} \times\left(2 \zeta+\frac{3}{2} \xi\right)+\frac{3}{r^{3}}\left[\left(\hat{\mathbf{n}} \cdot \mathbf{S}_{\mathbf{2}}\right)\left(\hat{\mathbf{n}} \times \mathbf{S}_{\mathbf{1}}\right)+\left(\hat{\mathbf{n}} \cdot \mathbf{S}_{\mathbf{1}}\right)\left(\hat{\mathbf{n}} \times \mathbf{S}_{\mathbf{2}}\right)\right]
$$

where $\mathbf{L}_{N} \equiv \mu \mathbf{x} \times \mathbf{v}$ denotes the Newtonian orbital angular momentum. Note that the precession of the spins is one order higher than the spins themselves. Therefore we may neglect this precession when evolving the equations to lowest order; Eq. (3) is only needed to verify that total angular momentum is conserved for the system, apart from that radiated away [11].

These equations of motion can be derived from a generalized Lagrangian which is a function of the relative position, velocity, and acceleration, given by

$$
\mathcal{L}=\frac{1}{2} \mu v^{2}+\mu \frac{m}{r}+\mathcal{L}_{S O}+\mathcal{L}_{S S}
$$

where

$$
\begin{gathered}
\mathcal{L}_{S O}=\frac{1}{2} \frac{\mu}{m}[\mathbf{v} \cdot(\mathbf{a} \times \xi)]+2 \frac{\mu}{r^{3}}\{\mathbf{v} \cdot[\mathbf{x} \times(\zeta+\xi)]\}, \\
\mathcal{L}_{S S}=\frac{1}{r^{3}}\left\{\left(\mathbf{S}_{\mathbf{1}} \cdot \mathbf{S}_{\mathbf{2}}\right)-3\left(\hat{\mathbf{n}} \cdot \mathbf{S}_{\mathbf{1}}\right)\left(\hat{\mathbf{n}} \cdot \mathbf{S}_{\mathbf{2}}\right)\right\} .
\end{gathered}
$$

Here the Euler-Lagrange equations are $\partial \mathcal{L} / \partial x^{i}-d p_{i} / d t=0$, where $p^{i}=\partial \mathcal{L} / \partial v^{i}-\dot{s}^{i}$, and $s^{i}=\partial \mathcal{L} / \partial a^{i}$. It is understood that wherever the acceleration appears in higher-order terms 
in the Euler-Lagrange equation, one substitutes the lower-order equation of motion (see [12] for discussion of acceleration-dependent Lagrangians).

The relative Lagrangian is invariant with respect to time translations so that there exists a conserved energy, given by $E=p^{i} v^{i}+s^{i} a^{i}-\mathcal{L}$. Evaluating this expression we obtain

$$
E=\frac{1}{2} \mu v^{2}-\mu \frac{m}{r}+E_{S O}+E_{S S}
$$

where

$$
\begin{gathered}
E_{S O}=\frac{\mu}{r^{2}}\{(\hat{\mathbf{n}} \times \mathbf{v}) \cdot \xi\}, \\
E_{S S}=\frac{1}{r^{3}}\left\{3\left(\hat{\mathbf{n}} \cdot \mathbf{S}_{\mathbf{1}}\right)\left(\hat{\mathbf{n}} \cdot \mathbf{S}_{\mathbf{2}}\right)-\left(\mathbf{S}_{\mathbf{1}} \cdot \mathbf{S}_{\mathbf{2}}\right)\right\} .
\end{gathered}
$$

We can define the total angular momentum as $\mathbf{L}=\zeta+(\mathbf{x} \times \mathbf{p})+(\mathbf{v} \times \mathbf{s})$, with the result

$$
\mathbf{L}=\zeta+\mathbf{L}_{N}+\mathbf{L}_{S O}
$$

where

$$
\mathbf{L}_{S O}=\frac{\mu}{m}\left\{\frac{m}{r}[\hat{\mathbf{n}} \times(\hat{\mathbf{n}} \times[2 \zeta+\xi])]-\frac{1}{2}[\mathbf{v} \times(\mathbf{v} \times \xi)]\right\}
$$

where there is no spin-spin contribution to L. Using the equations of motion and spin precession, Eqs. (11) and (3), it is straightforward to show explicitly that, to post-Newtonian order, $\dot{E}=\dot{\mathbf{L}}=0$.

\section{EVALUATION OF BDI MULTIPOLES}

The radiative energy loss of the system can be expressed in terms of symmetric and trace-free $(\mathrm{STF})$ radiative multipole moments (see [13] for a review). For the accuracy we require the energy loss rate is given by

$$
\frac{d E}{d t}=-\frac{1}{5}\left\{\stackrel{(3)}{I_{i j}} \stackrel{(3)}{I}_{i j}+\frac{5}{189} \stackrel{(4)}{I_{i j k}} \stackrel{(4)}{I}_{i j k}+\frac{16}{9} \stackrel{(3)}{J}_{i j} \stackrel{(3)}{J_{i j}}\right\}
$$


where $I_{i j}$ and $I_{i j k}$ are the STF "mass" quadrupole and octopole moments, and $J_{i j}$ is the STF "current" quadrupole moment, and $(n)$ over each moment denotes time derivatives.

We evaluate the radiative multipoles using the formalism developed by Blanchet, Damour and Iyer [5,6] (BDI). We restrict ourselves to the case of two well-separated, approximately spherically symmetric, rotating compact objects whose structure is given by that of a perfect fluid. Following our previous post-Newtonian approach [3, 14], we choose the following definition for the center of mass of each body

$$
\begin{gathered}
x_{A}^{i}=\frac{1}{m_{A}} \int_{A} x^{i} \rho^{*}(\mathbf{x})\left[1+\frac{1}{2} \bar{v}_{A}^{2}+\Pi-\frac{1}{2} \bar{U}_{A}\right] d^{3} x, \\
m_{A}=\int_{A} \rho^{*}(\mathbf{x})\left[1+\frac{1}{2} \bar{v}_{A}^{2}+\Pi-\frac{1}{2} \bar{U}_{A}\right] d^{3} x,
\end{gathered}
$$

where $\rho^{*}=\rho\left(1+\frac{1}{2} v^{2}+3 U\right)$ is the so-called "conserved density" [15], with $\rho$ the local mass density, $v$ the velocity, and $U$ the Newtonian gravitational potential; $\Pi$ is the specific internal energy density, $\bar{v}_{A}^{i}=v^{i}-v_{A}^{i}, v_{A}^{i}=d x_{A}^{i} / d t$, and $\bar{U}_{A}$ is the Newtonian potential produced by the $A$-th body itself.

However, when spin effects are to be included, there is a subtle difference between the center of mass defined above, and the center of mass used in the equations of moton (D). The latter defines the center of mass world line $x_{A}{ }^{\mu}$ of each body using a so-called "spin supplementary condition" (SSC), given by $S_{A}{ }^{\mu \nu} u_{A \nu}=0$, where $u_{A}^{\mu}$ is the four-velocity of the center-of-mass world line, and

$$
S_{A}^{\mu \nu} \equiv 2 \int\left(x^{[\mu}-x_{A}^{[\mu}\right) \tau^{\nu] 0} d^{3} x,
$$

where $\tau^{\mu \nu}$ denotes the stress-energy tensor of matter plus gravitational fields, satisfying $\tau_{, \nu}^{\mu \nu}=0$, and square brackets around indices denote antisymmetrization. Note that the spin $\mathbf{S}$ of each body is defined by $S_{A}^{i}=\frac{1}{2} \epsilon_{i j k} S_{A}^{j k}$.

It is then simple to show that, evaluating the BDI multipoles using the center of mass definition Eq. (11a), and then making the transformation 


$$
x_{A}^{i} \longrightarrow x_{A}^{i}+\frac{1}{2 m_{A}}\left(\mathbf{v}_{\mathbf{A}} \times \mathbf{S}_{\mathbf{A}}\right)^{i}
$$

we can convert all expressions to the center of mass defined by the SSC of the equations of motion. Because the correction is of post-Newtonian order, it needs to be made only in the Newtonian-order multipoles.

A useful check of the consistency of our approach comes from evaluating the mass dipole moment of the system, given by Eq. (A16b) of Ref. [6], and making the transformation

(13). The result is $I^{i}=\sum_{A}\left\{m_{A} x_{A}^{i}+\left(\mathbf{v}_{\mathbf{A}} \times \mathbf{S}_{\mathbf{A}}\right)^{i}\right\}$. The original two-body equations of motion from which Eq. (1) is derived [8] then imply that $\ddot{I}^{i}=0$, as expected (uniform motion of the system's center of mass).

Evaluating the other multipoles, transforming them to the SSC of our equations of motion using Eq. (13), and then transforming them into relative coordinates, we obtain

$$
\begin{gathered}
I^{i j}=\mu\left(x^{i} x^{j}\right)^{S T F}+\frac{8}{3} \eta\left[x^{i}(\mathbf{v} \times \xi)^{j}\right]^{S T F}-\frac{4}{3} \eta\left[v^{i}(\mathbf{x} \times \xi)^{j}\right]^{S T F}, \\
I^{i j k}=\mu\left(x^{i} x^{j} x^{k}\right)^{S T F}, \\
J^{i j}=-\mu\left(\frac{\delta m}{m}\right)\left[x^{i}(\mathbf{x} \times \mathbf{v})^{j}\right]^{S T F}+\frac{3}{2}\left(x^{i} \sigma^{j}\right)^{S T F},
\end{gathered}
$$

where $\delta m=m_{1}-m_{2}, \eta=\mu / m$, and $\sigma=\left(m_{2} / m\right) \mathbf{S}_{\mathbf{1}}-\left(m_{1} / m\right) \mathbf{S}_{\mathbf{2}}$. Note that, to postNewtonian order, there are no explicit spin-spin contributions to the multipoles.

\section{ENERGY LOSS AND INSPIRAL OF CIRCULAR ORBITS}

Taking time derivatives of Eqs. (14), substituting the equations of motion where appropriate, and substituting the results into Eq. (10), we obtain the energy loss rate

$$
\begin{aligned}
\frac{d E}{d t}= & -\frac{8}{15} \frac{m^{2} \mu^{2}}{r^{4}}\left\{12 v^{2}-11 \dot{r}^{2}\right. \\
& +\frac{\hat{\mathbf{n}} \times \mathbf{v}}{m r} \cdot\left[\zeta\left(27 \dot{r}^{2}-37 v^{2}-12 \frac{m}{r}\right)+\xi\left(51 \dot{r}^{2}-43 v^{2}+4 \frac{m}{r}\right)\right] \\
& +\frac{1}{2 m \mu r^{2}}\left[3\left(\mathbf{S}_{\mathbf{1}} \cdot \mathbf{S}_{\mathbf{2}}\right)\left(47 v^{2}-55 \dot{r}^{2}\right)-3\left(\hat{\mathbf{n}} \cdot \mathbf{S}_{\mathbf{1}} \hat{\mathbf{n}} \cdot \mathbf{S}_{\mathbf{2}}\right)\left(168 v^{2}-269 \dot{r}^{2}\right)\right. \\
& \left.\left.+71\left(\mathbf{v} \cdot \mathbf{S}_{\mathbf{1}} \mathbf{v} \cdot \mathbf{S}_{\mathbf{2}}\right)-171 \dot{r}\left(\mathbf{v} \cdot \mathbf{S}_{\mathbf{1}} \hat{\mathbf{n}} \cdot \mathbf{S}_{\mathbf{2}}+\hat{\mathbf{n}} \cdot \mathbf{S}_{\mathbf{1}} \mathbf{v} \cdot \mathbf{S}_{\mathbf{2}}\right)\right]\right\} .
\end{aligned}
$$


We now restrict ourselves to the case of nearly circular orbits, by which we mean orbits whose inspiral timescale due to gravitational radiation energy loss is long compared to an orbital timescale, and in which $r=$ constant + small, periodic perturbations due to spin couplings (when the spins are orthogonal to the orbital plane, the spin perturbations are constant). To simplify the discussion, we then take an angular average of all quantities over an orbit. We then have $\ddot{r}=\dot{r}=\mathbf{n} \cdot \mathbf{v}=0, v^{2}=r^{2} \Omega^{2}$, where $\Omega$ is the angular frequency, $\mathbf{L}_{\mathbf{N}}=\mu r^{2} \Omega \hat{\mathbf{L}}$, where $\hat{\mathbf{L}}$ is a unit vector orthogonal to the orbital plane. The equation $\hat{\mathbf{n}} \cdot \mathbf{a}=\ddot{r}-r \Omega^{2}$ yields the following orbit-averaged relationship for a circular orbit

$$
r=\Omega^{-2 / 3} m^{1 / 3}\left\{1-\frac{1}{3} \frac{\Omega}{m} \hat{\mathbf{L}} \cdot(2 \zeta+3 \xi)-\frac{1}{2} \frac{\Omega^{4 / 3}}{\mu m^{5 / 3}}\left(\mathbf{S}_{\mathbf{1}} \cdot \mathbf{S}_{\mathbf{2}}-3 \hat{\mathbf{L}} \cdot \mathbf{S}_{\mathbf{1}} \hat{\mathbf{L}} \cdot \mathbf{S}_{\mathbf{2}}\right)\right\} .
$$

These conditions lead to the following results for the energy and energy loss rate for a circular orbit, averaged over an orbit, expressed in terms of $\Omega$ :

$$
\begin{aligned}
E(\Omega)=-\frac{1}{2} \mu(m \Omega)^{2 / 3} & \left\{1+\frac{\Omega}{m} \hat{\mathbf{L}} \cdot\left(\frac{8}{3} \zeta+2 \xi\right)+\frac{\Omega^{4 / 3}}{\mu m^{5 / 3}}\left(\mathbf{S}_{\mathbf{1}} \cdot \mathbf{S}_{\mathbf{2}}-3 \hat{\mathbf{L}} \cdot \mathbf{S}_{\mathbf{1}} \hat{\mathbf{L}} \cdot \mathbf{S}_{\mathbf{2}}\right)\right\} \\
\frac{d E}{d t}(\Omega)= & -\frac{32}{5} \eta^{2}(m \Omega)^{10 / 3}\left\{1-\frac{1}{4} \frac{\Omega}{m} \hat{\mathbf{L}} \cdot(11 \zeta+5 \xi)\right. \\
& \left.-\frac{1}{48} \frac{\Omega^{4 / 3}}{\mu m^{5 / 3}}\left(103 \mathbf{S}_{\mathbf{1}} \cdot \mathbf{S}_{\mathbf{2}}-289 \hat{\mathbf{L}} \cdot \mathbf{S}_{\mathbf{1}} \hat{\mathbf{L}} \cdot \mathbf{S}_{\mathbf{2}}\right)\right\}
\end{aligned}
$$

The result for the evolution of orbital frequency is

$$
\begin{aligned}
\frac{\Omega^{2}}{\dot{\Omega}}= & \frac{\Omega^{2} d E / d \Omega}{d E / d t}=\frac{5}{96} \frac{m}{\mu}(m \Omega)^{-5 / 3}\left\{1+\frac{\Omega}{m} \hat{\mathbf{L}} \cdot\left(\frac{113}{12} \zeta+\frac{25}{4} \xi\right)\right. \\
& \left.+\frac{1}{48} \frac{\Omega^{4 / 3}}{\mu m^{5 / 3}}\left(247 \mathbf{S}_{\mathbf{1}} \cdot \mathbf{S}_{\mathbf{2}}-721 \hat{\mathbf{L}} \cdot \mathbf{S}_{\mathbf{1}} \hat{\mathbf{L}} \cdot \mathbf{S}_{\mathbf{2}}\right)\right\}
\end{aligned}
$$

The energy and loss rate can also be expressed exclusively in terms of $r$ using Eq. (16), and an equation for the rate of inspiral obtained. The result is

$$
\begin{aligned}
\dot{r}= & -\frac{64}{5} \eta\left(\frac{m}{r}\right)^{3}\left\{1-\frac{7}{12} \frac{(m / r)^{3 / 2}}{m^{2}} \hat{\mathbf{L}} \cdot(19 \zeta+15 \xi)\right. \\
& \left.-\frac{5}{48} \frac{(m / r)^{2}}{\mu m^{3}}\left(59 \mathbf{S}_{\mathbf{1}} \cdot \mathbf{S}_{\mathbf{2}}-173 \hat{\mathbf{L}} \cdot \mathbf{S}_{\mathbf{1}} \hat{\mathbf{L}} \cdot \mathbf{S}_{\mathbf{2}}\right)\right\} .
\end{aligned}
$$




\section{DISCUSSION OF RESULTS}

A signal template whose frequency evolution is given by Eq. (18) can be said to match the signal if the accumulated phase in GW from the time the signal enters the detectors' sensitive bandwidth to the time it leaves it differs from that of the template by less than $2 \pi$ radians. Thus one can obtain a crude estimate of the accuracy with which a parameter characterizing the template can be determined by finding that change in the parameter that leads to a change of $2 \pi$ in the accumulated phase [2]. Such estimates are only crude and probably optimistic, because they do not take into account signal-to-noise issues or correlations among multiple parameters.

The accumulated phase in gravitational waves is given by

$$
\Phi_{G W} \equiv 2 \pi \int_{t_{i}}^{t_{f}} f d t=2 \int_{\Omega_{i}}^{\Omega_{f}}\left(\Omega^{2} / \dot{\Omega}\right) d \Omega / \Omega
$$

where $f=\Omega / \pi$ is the GW signal frequency. Integrating the SO and SS terms in Eq. (18), and ignoring precession of the spins or of the orbital angular momentum, we can estimate the accuracy of determination of the spin parameters. For example, for two equal-mass neutron stars, we find, for the spin-orbit terms,

$$
\frac{\Delta \zeta_{z}}{\zeta_{z}} \simeq 0.38\left(\frac{m}{2.8 M_{\odot}}\right)^{5 / 3}\left(\frac{f_{\mathrm{in}}}{30 \mathrm{~Hz}}\right)^{2 / 3}\left(\frac{10 \mathrm{~km}}{r_{N S}}\right)^{2}\left(\frac{P_{N S}}{10 \mathrm{~ms}}\right)
$$

where $r_{N S}$ and $P_{N S}$ are the neutron-star radius and rotation period (suitably averaged over the two stars), and $f_{\text {in }}$ is the GW frequency entering the detector bandwidth. For a $10 M_{\odot}$ black hole with spin $S_{1} \equiv m_{1} a_{1}$, where $a_{1}<m_{1}$ is the Kerr parameter, and a $1.4 M_{\odot}$ neutron star, the quantity $a_{1} / m_{1}$ be determined to an error $\Delta\left(a_{1} / m_{1}\right) \simeq 0.016$ for $a_{1} / m_{1}>0.03$, while for $a_{1} / m_{1} \ll 0.03$, the spin of the companion neutron star cannot be found to better than a factor of four, for a $10 \mathrm{~ms}$ rotation period. For two $10 M_{\odot}$ black holes, the error in the net "Kerr parameter per mass" of the system projected orthogonal to the orbital plane, $\Delta\left(a_{1} / m_{1}+a_{2} / m_{2}\right)_{z}$, can be estimated to be about \pm 0.1 . However, Monte-Carlo studies indicate that strong correlations between post-Newtonian terms (dependent on $\mu$ ) and spin-orbit terms are likely to weaken these estimates substantially [2]. 
Spin-spin terms, on the other hand, have negligible effect on the accumulated phase for most systems of interest. Only for two extreme Kerr black holes are these terms discernable; for two $10 M_{\odot}$ black holes with both spins orthogonal to the orbital plane, we estimate $\Delta\left(S_{1} S_{2}\right) /\left|S_{1} S_{2}\right| \simeq 0.4$

\section{ACKNOWLEDGMENTS}

We are grateful to Kip Thorne for sharing his early calculations of spin effects with us and for encouraging us to pursue this problem vigorously. We also acknowledge useful discussions with Sam Finn. This research is supported in part by the National Science Foundation Grant No. 89-22140. 


\section{REFERENCES}

[1] K. S. Thorne, in 300 Years of Gravitation (Cambridge University Press, Cambridge, 1987), p.330; B. S. Sathyaprakash and S. V. Dhurandhar, Phys. Rev. D 44, 3819 (1991); K. Jotania, S. M. Wagh and S. V. Dhurandhar, Phys. Rev. D 46, 2507 (1992); L. S. Finn, Phys. Rev. D., in press.

[2] C. Cutler, T. A. Apostolatos, L. Bildsten, L. S. Finn, E. E. Flanagan, D. Kennefick, D. M. Markovic, A. Ori, E. Poisson, G. J. Sussman, and K. S. Thorne, Caltech Preprint No. GRP-316 (1992).

[3] R. V. Wagoner and C. M. Will, Astrophys. J. 210, 764 (1976); 215, 984 (1977).

[4] C. W. Lincoln and C. M. Will, Phys. Rev. D 42, 1123 (1990).

[5] L. Blanchet and T. Damour, Ann. Inst. Henri Poincaré A, 50, 377 (1989).

[6] T. Damour and B. R. Iyer, Ann. Inst. Henri Poincaré 54, 115 (1991).

[7] B. M. Barker and R. F. O'Connell, Gen. Relativ. Gravit. 11, 149 (1979).

[8] For a thorough review, see T. Damour, in 300 Years of Gravitation (Cambridge University Press, Cambridge, 1987), p.128.

[9] V. A. Brumberg, Essential Relativistic Celestial Mechanics (Adam Hilger, Bristol, 1991), p. 155.

[10] L. Bildsten and C. Cutler, Astrophys. J., in press.

[11] Over many orbits, precession of the spins and the orbital plans can have significant modulating effects on gravitational waveforms; see G. J. Sussman and K. S. Thorne, in preparation.

[12] T. Damour and N. Deruelle, C. R. Acad. Sci. Paris 293, 537 (1981); 293, 877 (1981); T. Damour, in Gravitational Radiation, edited by N. Deruelle and T. Piran (North Holland, Amsterdam, 1983), p. 59; L. P. Grishchuk and S. M. Kopejkin, in Relativity 
in Celestial Mechanics and Astrometry, edited by J. Kovalevsky and V. A. Brumberg (Reidel, Dordrecht, 1986), p. 19.

[13] K. S. Thorne, Rev. Mod. Phys. 52, 299 (1980).

[14] A. G. Wiseman, Phys. Rev. D 46, 1517 (1992).

[15] See, for example, C. M. Will, Theory and Experiment in Gravitational Physics (Cambridge University Press, Cambridge, 1981), p. 111. 